\title{
Nocardia nova causing empyema necessitatis after lung re-transplantation: a case report
}

\author{
Cecília Bittencourt Severo ${ }^{[1],[2], ~ L e t i ́ c i a ~ B e a t r i z ~ M a t t e r ~}{ }^{[3],[4]}$, Flávio de Mattos Oliveira ${ }^{[1]}$, \\ Agueda Palmira Castagna Vargas ${ }^{[4]}$, Sadi Marcelo Schio ${ }^{[5]}$, José de Jesus Peixoto Camargo ${ }^{[5]}$, \\ Bruno Hochhegger ${ }^{[2],[6]}$ and Luiz Carlos Severo ${ }^{[1],[7]}$
}

[1]. Laboratório de Micologia, Hospital Santa Rita, Irmandade Santa Casa de Misericórdia de Porto Alegre, Porto Alegre, Rio Grande do Sul, Brasil. [2]. Departamento de Ciências Básicas, Universidade Federal de Ciências da Saúde de Porto Alegre, Porto Alegre, Rio Grande do Sul, Brasil.

[3]. Departamento de Ciências da Saúde, Universidade Regional Integrada do Alto Uruguai e das Missões, Santo Ângelo, Rio Grande do Sul, Brasil.

[4]. Departamento de Medicina Veterinária Preventiva, Universidade Federal de Santa Maria, Santa Maria, Rio Grande do Sul, Brasil.

[5]. Serviço de Transplante Pulmonar, Irmandade Santa Casa de Misericórdia de Porto Alegre, Porto Alegre, Rio Grande do Sul, Brasil.

[6]. Serviço de Radiologia, Irmandade Santa Casa de Misericórdia de Porto Alegre, Porto Alegre, Rio Grande do Sul, Brasil.

[7]. Departamento de Medicina Interna, Universidade Federal do Rio Grande do Sul, Porto Alegre, Rio Grande do Sul, Brasil.

\begin{abstract}
We report herein a case of thoracic infection due to Nocardia nova following lung re-transplantation performed for emphysema related to alpha-1-antitrypsin deficiency. The infection extended from the lung into the pleural space, thoracic wall, and mediastinum, presenting as pericarditis and empyema necessitatis. Nocardia nova was identified by $16 \mathrm{~S}$ ribosomal deoxyribonucleic acid (rDNA) sequencing and phylogenetic analysis. According to a literature search of PubMed, LILACS and MEDLINE databases, we describe herein the first case of empyema necessitatis caused by $N$. nova species in a transplanted patient.
\end{abstract}

Keywords: Nocardia nova. Pericarditis. Empyema necessitatis.

\section{INTRODUCTION}

Empyema necessitatis refers to inflammatory tissue that extends directly from the pleural cavity into the thoracic wall, forming a mass (a cold abscess requiring drainage) in the extra-pleural soft tissues. It is a very rare complication of different infectious etiologies; tuberculosis and actinomycosis are the most common causative organisms ${ }^{(1)}$. Recently, cases of nocardiosis with chest wall extension ${ }^{(2)}$ and $N$. asteroides empyema necessitatis have been observed ${ }^{(3)}$. Herein, we report the first case of empyema necessitatis caused by $N$. nova.

\section{CASE REPORT}

A 50-year-old woman underwent lung re-transplantation in July 2011, after developing chronic lung allograft dysfunction. The patient had a long history of emphysema secondary to alpha-1-antitrypsin deficiency. She was subjected to doublelung volume-reduction surgery in 2003 and single-lung transplantation in 2006. The lung re-transplantation in 2011 was bilateral. Her immunosuppressive regimen consisted of prednisone, mycophenolate, and tacrolimus. Five months

Corresponding author: Dr. Luiz Carlos Severo.

e-mail: severo@santacasa.tche.br

Received 11 November 2015

Accepted 5 April 2016 after re-transplantation, the patient presented to the transplant clinic with dyspnea. Bronchoscopy identified stenosis of the left bronchial anastomosis. Endoscopic attempts to manage the stenosis failed and a lower left lobectomy was performed.

One month later, she presented to the clinic with dyspnea and a painful draining mass located on her left lateral chest wall. On examination, she was afebrile and an external fistula was identified in the left inframammary region. The patient received intravenous ceftriaxone as empiric antimicrobial therapy for subcutaneous infection, with consequent clinical improvement. However, when the therapy was stopped, the problem worsened and the chest radiograph revealed a pleural effusion.

In the investigation, the pleural fluid consisted of a purulent exudate (Figure 1A). Gram-positive filamentous bacilli (Figure 1B) were detected in the exudate. These bacilli were decolorized by Ziehl-Neelsen staining. A contrast-enhanced axial computed tomography (CT) scan with a mediastinal window demonstrated atelectasis in the left lower lobe, associated with bilateral pleural and pericardial effusions. In addition, pleural and pericardial thickening was revealed, suggesting a complicated effusion. The clinical and cytological findings were suggestive of actinomycosis. The patient was thus treated with penicillin, but failed to show any clinical improvement. Kinyoun staining was performed and revealed branching, weakly acid-fast, filaments (Figure 1C). Aerobic culture of the pleural fluid suggested Nocardia. 


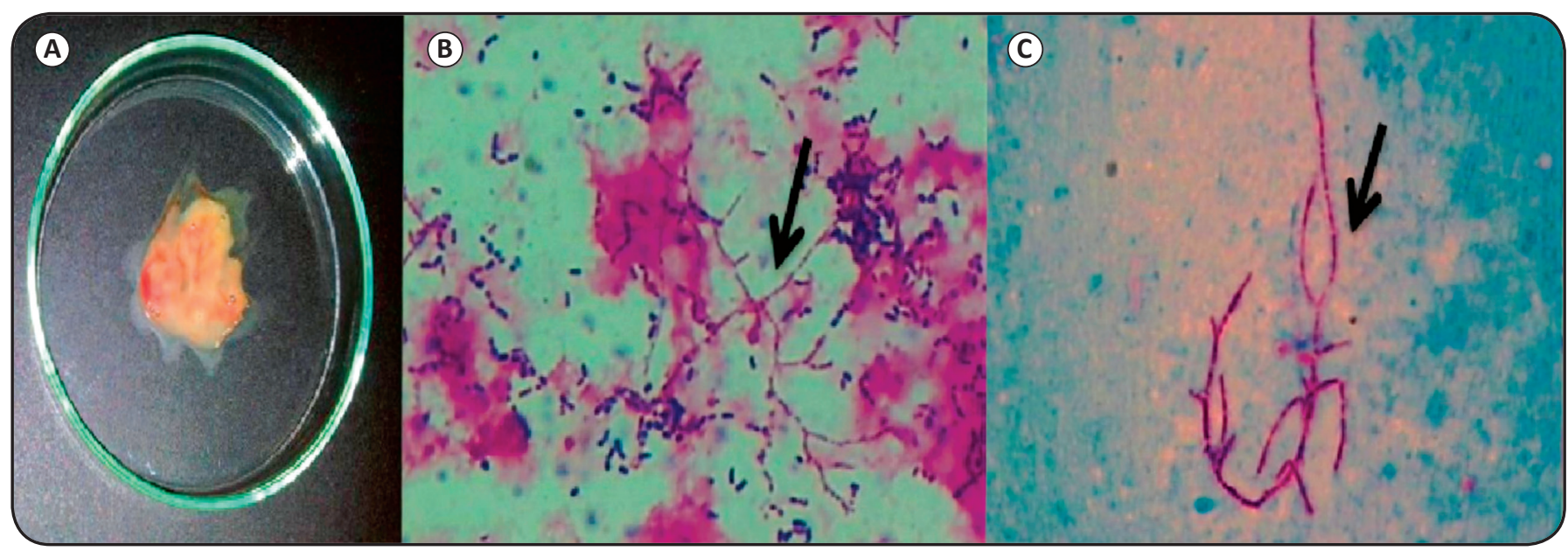

FIGURE 1. Nocardia nova pictures. (A) Purulent pleural fluid obtained via aspiration of the pleural cavity. (B) Staining performed directly from the pleural effusion showing Gram-positive rod-shaped bacteria. (C) Partially acid-fast branching filaments showed by Kinyoun Staining. Note: Figures B and C were observed by microscopy at 1,000-times magnification.

In the diagnosis, deoxyribonucleic acid (DNA) from isolated colonies (SBP72a/12: sampled from pleural fluid; and SBP72b/12 sampled from the pleural cavity secretion) was isolated and molecular diagnosis was performed by analyzing the partial sequence (approximately 1,300 base pairs) of $16 \mathrm{~S}$ ribosomal ribonucleic acid (rRNA) gene amplified with universal primers ${ }^{(4)}$. Polymerase chain reaction (PCR) products were sequenced by ACTGene Análises Moleculares Ltda [Centro de Biotecnologia, Universidade Federal do Rio Grande do Sul (UFRGS), Porto Alegre, RS, Brazil] using the automatic sequencer ABI-PRISM ${ }^{\circledR} 3100$ Genetic Analyzer (Applied Biosystems). Consensus sequences were assembled using Staden Package 4. Figure 2 shows the strong homology of the samples with others $N$. nova strains and the relationship with others species available in GenBank dataset. The partial 16S rRNA gene sequences of SBP72a/12 and SBP72b/12 were deposited in the GenBank database.

In the follow-up, $N$. nova was identified, the patient received treatment with intravenous trimethoprim/sulfamethoxazole (TMP/SMX) 320/1,600mg every six hours for one month, with success. Central nervous system disease was ruled out. Finally, the patient was discharged on oral TMP/SMX (160/800 BID). At discharge, she was asymptomatic, and the lesions had improved both clinically and radiologically. At the time of her last followup visit, five months after discontinuation of antimicrobial therapy, she remained well.

\section{DIscussion}

Traditional identification of Nocardia to species level by phenotyping is cumbersome and the results are sometimes difficult to interpret. The recent introduction of molecular methods has had an enormous impact on the taxonomy of Nocardia and has resulted in the recognition of numerous new species. Worldwide, respiratory and disseminated infections are most often due to members of the previously broadly defined $N$. asteroides complex, which contains three strains: Nocardia asteroides sensu stricto, Nocardia farcinica, and N. nova complex. The latter includes $N$. nova species ${ }^{(5)}$.

In a search of PubMed/MEDLINE and LILACS databases, we found no previous report of empyema necessitatis caused by $N$. nova. The key words used for the search were Nocardia nova, nocardiosis, lung transplant, and empyema necessitatis, and it was performed until September 2015. We describe here the first case of empyema necessitatis caused by $N$. nova species in a transplanted patient.

A contemporary case-control study found that the incidence of $N$. nova isolated from solid organ transplant recipients was higher than $N$. farcinica ( $49 \%$ vs. $28 \%$, respectively $)^{(6)}$. In a review of 17 patients with an underlying malignancy and Nocardia bacteremia, $N$. nova complex was demonstrated to be responsible for $35 \%$ of the cases. Bacterial adhesion to intravenous catheters may play a role in the incidence of intravascular $N$. nova infection ${ }^{(7)(8)}$.

Infection of the lung parenchyma and pleural space by $N$. nova has previously been observed ${ }^{(9)(10)(11)}$. However, direct extension from the lung into the pleural cavity, progressing to penetration of the mediastinum, with consequent pericarditis and empyema necessitates-specifically in a lung-transplant recipient - has not previously been reported. Physicians should be aware of this possibility in order to obtain and submit appropriate thoracic specimens to the laboratory to optimize the recovery of this microorganism.

In conclusion, we advocate maintaining a high index of suspicion for nocardiosis in a patient with underlying immunosuppression, and instituting an active approach to obtaining specimens for diagnostic studies. Opportunistic pathogens such as observed in this case, may be weakly acidfast and will be decolorized by the Ziehl-Neelsen procedure. Previously diagnosed as "Nocardia asteroides complex", routine microbiology laboratories should attempt to identify the organism to species level. Finally, clinicians should be aware that $N$. nova could be associated with empyema necessitatis. 


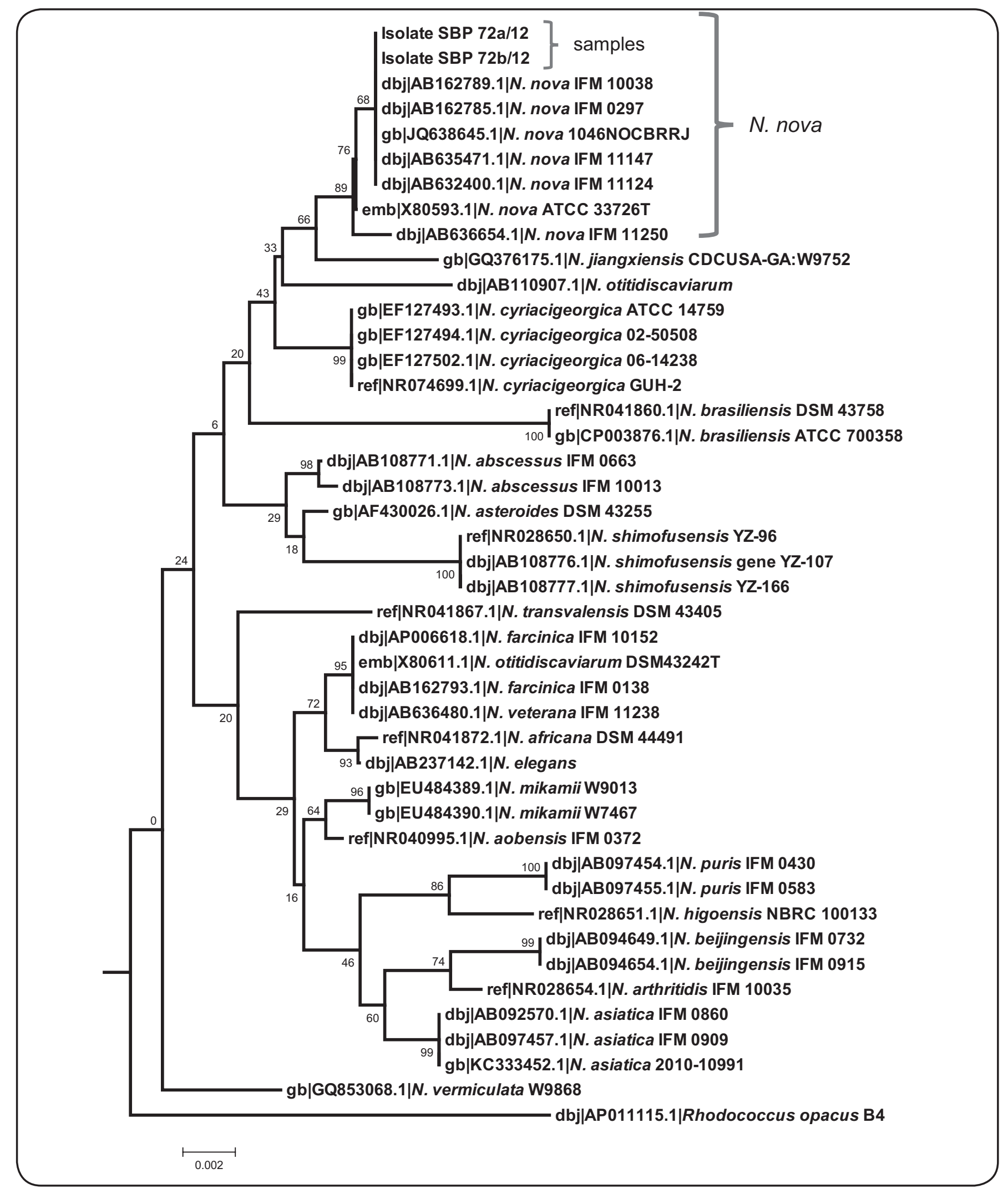

FIGURE 2. Nucleotide sequence analysis. Phylogenetic relationship derived from $16 \mathrm{~S}$ rDNA sequences showing the homology between Nocardia nova (samples SBP 72a/12 and SBP 72b/12) and 16S rDNA sequences of other Nocardia species. The phylogenetic tree was built in the MEGA 5.2 software program, using the Neighbor-Joining algorithm and the Tamura 3-parameter substitution model. Rhodococcus opacus B4 (AP011115.1) was used as the out group reference. The percentage of replicate trees in which the associated taxa clustered together in the bootstrap test (1,000 replicates) is shown next to each branch. SBP72a/12 was isolated from pleural fluid and SBP72b/12 was isolated from the pleural cavity. N: Nocardia; rDNA: ribosomal deoxyribonucleic acid; SBP: Setor de Bacteriologia e Pesquisa; MEGA: Molecular Evolutionary Genetics Analysis. 


\section{Acknowledgements}

The authors acknowledge the Coordenação de Aperfeiçoamento de Pessoal de Nivel Superior (CAPES) for the Letícia B. Matter's Post-doctorate scholarship.

\section{Financial Support}

Programa Nacional de Pós-Doutorado/Coordenação de Aperfeiçoamento de Pessoal de Nivel Superior Project number 2734/2011.

\section{REFERENCES}

1. Reyes CV, Thompson KS, Jensen J. Fine needle aspiration biopsy of masititis secondary to empyema necessitatis. A report of two cases. Acta Cytol 1999; 43:873-876.

2. Kanne JP, Yandow DR, Mohammed TL, Meyer CA. CT findings of pulmonary nocardiosis. AJR Am J Roentgenol 2011; 197:266-272.

3. Cadena J, Cadena A, Hallmark K, Ahuja SK. Pulmonary nocardiosis mimicking empyema necessitatis. J Resp Dis 2008; 29:1-6.

4. Fredricks DN, Relman DA. Improved amplification of microbial DNA from blood cultures by removal of the PCR inhibitor sodium polyanetholesulfonate. J Clin Microbiol 1998; 36:2810-2816.
5. Brown-Elliott BA, Brown JM, Conville PS, Wallace Jr RJ. Clinical and laboratory features of the Nocardia spp. based on current molecular taxonomy. Clin Microbiol Rev 2006; 19:259-282.

6. Peleg AY, Husain S, Qureshi ZA, Silveira FP, Sarumi M, Shutt $\mathrm{KA}$, et al. Risk factors, clinical characteristics, and outcome of Nocardia infection in organ transplant recipients: a matched casecontrol study. Clin Infect Dis 2007; 44:1307-1314.

7. Al Akhrass F, Hachem R, Mohamed JA, Tarrand J, Kontoyiannis DP, Chandra J, et al. Central venous catheter-associated Nocardia bacteremia in cancer patients. Emerg Infect Dis 2011; 17:1651-1658.

8. Gomila BS, Sabater SV, Merino JP, Igual RA, Moreno RM. Abscesso cerebral por Nocardia nova. Rev Chil Infect 2012; 29:112-113.

9. Burucoa C, Breton I, Ramassamy A, Soyer J, Becq-Giraudon B, Fauchère JL. Western blot monitoring of disseminated Nocardia nova infection treated with clarithromycin, imipenem, and surgical drainage. Eur J Clin Microbiol Infect Dis 1996; 15: 943-947.

10. Couraud S, Houot R, Coudurier M, Ravel AC, Coiffier B, Souquet PJ. Nocardial pulmonary infection. Rev Mal Respir 2007; 24: 353-357.

11. Tan CK, Lai CC, Lin SH, Liao CH, Chou CH, Hsu HL, et al. Clinical and microbiological characteristics of nocardiosis including those caused by emerging Nocardia species in Taiwan, 1998-2008. Clin Microbiol Infect 2010; 16:966-972. 